\title{
Alterstice
}

Revue internationale de la recherche interculturelle

International Journal of Intercultural Research

Revista International de la Investigacion Intercultural

\section{Des clubs de sciences parascolaires : un outil pour développer des liens chez des jeunes de diverses communautés ethniques et pour appuyer un apprentissage transformationnel chez les élèves et les futurs enseignants [synthèse en français]}

Jrène Rahm, Annie Malo et Michel Lepage

Volume 6, numéro 1, 2016

Prendre en compte la diversité à l'école

URI : https://id.erudit.org/iderudit/1038278ar

DOI : https://doi.org/10.7202/1038278ar

Aller au sommaire du numéro

Éditeur(s)

Alterstice

ISSN

1923-919X (numérique)

Découvrir la revue

Citer ce document

Rahm, J., Malo, A. \& Lepage, M. (2016). Des clubs de sciences parascolaires : un outil pour développer des liens chez des jeunes de diverses communautés ethniques et pour appuyer un apprentissage transformationnel chez les élèves et les futurs enseignants [synthèse en français]. Alterstice, 6(1), 53-55.

https://doi.org/10.7202/1038278ar 


\title{
Des clubs de sciences parascolaires : un outil pour développer des liens chez des jeunes de diverses communautés ethniques et pour appuyer un apprentissage transformationnel chez les élèves et les futurs enseignants [synthèse en français*]
}

\author{
Jrène Rahm ${ }^{1}$, Annie Malo ${ }^{1}$ et Michel Lepage ${ }^{1}$
}

\author{
Rattachement des auteurs \\ ${ }^{1}$ Université de Montréal, Montréal, Canada.
}

\section{Correspondance}

jrene.rahm@umontreal.ca

\section{Mots clés}

clubs de science parascolaires, jeunes immigrants, formation en enseignement

\section{Pour citer cet article}

Rahm, J., Malo, A. et Lepage, M. (2016). Des clubs de sciences parascolaires: un outil pour développer des liens chez des jeunes de diverses communautés ethniques et pour appuyer un apprentissage transformationnel chez les élèves et les futurs enseignants [synthèse en français]. Alterstice, 6(1), 53-56. [Youth voice driven afterschool science clubs: A tool to develop new alliances in ethnically diverse communities in support of transformative learning for preservice teachers and youth]

*L'article complet en anglais est disponible sur le site d'Alterstice.

Plusieurs recherches ont déjà étudié les bienfaits des activités parascolaires en général, et plus particulièrement des clubs scientifiques, pour amener les jeunes immigrants à mobiliser leurs ressources et leurs connaissances en vue de transformer leur façon d'apprendre (Tan et Calabrese Barton, 2012). Ces activités parascolaires sont devenues aussi des lieux importants d'immersion pour les futurs enseignants, les mettant en contact avec la vie des communautés immigrantes et les encourageant à connaître plus à fond les situations de ces dernières, ce qui contribue à créer de riches situations d'apprentissage pour leur formation d'enseignants (McDonald, Bowman et Brayko, 2013; Murrell, 2000). Toutefois, peu d'études se sont intéressées aux effets de la participation des jeunes immigrants et des futurs enseignants à des clubs de sciences parascolaires en milieu urbain. Dans le contexte de leur formation en enseignement, les étudiants sont habituellement tenus à l'écart des réalités culturelles des milieux où ils seront appelés à travailler (Zeichner, 2010a et 2010b). Cet article présente les résultats d'une recherche-action qui s'est déroulée dans trois écoles secondaires de Montréal desservant une population d'élèves d’origines diverses et composée à $80 \%$ d'immigrants de première et de deuxième générations.

S'appuyant sur les principes de l'apprentissage transformationnel et sur une vision communautaire de la formation des enseignants, les clubs de science avaient pour but de répondre aux besoins et aux intérêts des jeunes élèves envers des activités scientifiques et d'intégrer les expériences culturelles diverses que ces jeunes participants 
peuvent y apporter. L'engagement personnel de chacun était encouragé, dans un désir de création et d'échanges basé sur un dialogue bilingue et biculturel respectant les multiples trajectoires des élèves et des participants universitaires (Gutiérrez, 2014). Pour ce faire, l'équipe de recherche a convenu d'encourager la production par les jeunes de vidéos scientifiques à caractère documentaire et destinées à leurs pairs. En lien avec les ressources communautaires, les participants avaient aussi accès à des séjours - en cours d'année scolaire et durant l'été - au Jardin botanique et à un camp d'ingénierie ainsi qu'à des visites aux musées. Pour les étudiants en formation à l'enseignement, l'expérience visait à apporter une connaissance plus approfondie de la réalité hors de l'école vécue par des communautés au sein desquelles ils seront appelés à enseigner, participant ainsi à un modèle de formation axé sur l'implication communautaire et sur une conciliation théorie-pratique.

Un groupe de 12 élèves de 13 et 14 ans ont participé aux activités du club de science. Trois d'entre eux sont nés au Canada, les autres sont issus d'une immigration récente. Leurs origines sont diverses : Algérie, Arabie saoudite, Cambodge Maroc, Sénégal, Tunisie et Vietnam. Les données recueillies proviennent, d'une part, des documents vidéos réalisés et, d'autre part, d'une entrevue avec un étudiant en formation à l'enseignement qui a agi à titre d'animateur dans un des clubs ainsi que des réflexions de 25 étudiants en enseignement analysées à la suite de leurs visites au club.

Au total, cinq documentaires ont été produits par les élèves sur des sujets qu'ils avaient eux-mêmes choisis :

- les grenouilles,

- l'extinction de certaines espèces,

- le monde canin et les prédateurs,

- la technologie,

- les effets de la caféine sur le corps humain.

Les entretiens ont révélé que les participants ont beaucoup apprécié le fait de pouvoir réaliser leurs films comme ils l'entendaient, développant ainsi des habiletés technologiques, et aussi de pouvoir discuter avec les experts qui ont accepté de les conseiller. Les séjours dans les camps et au Jardin botanique ont contribué à éveiller davantage leur curiosité et leur intérêt, élément important de l'apprentissage transformationnel envisagé : réalisation de projets personnels, échanges positifs et respectueux avec des pairs, présence des insectes et des fleurs dans l'environnement naturel. Pour la plupart d'entre eux, il s'agissait de leur première expérience de contact avec le monde des sciences hors de l'école.

L'analyse des réflexions des étudiants en enseignement au sujet du club de sciences parascolaire a montré que cette expérience leur a permis de découvrir un milieu d'enseignement différent, au sein d'une école multiethnique que plusieurs n'avaient jamais eu l'occasion de côtoyer. Une expérience qui a notoirement contribué à ébranler leurs préjugés et à offrir un nouveau sens à leur formation :

Quand je suis entré dans l'école, je me sentais comme un étranger... Pourtant, les élèves m'ont accueilli très amicalement... J'ai vu des élèves souriants, blaguant entre eux. Je m'attendais à voir une école pauvre et misérable, à l'image de certains secteurs du quartier. Ce que j'ai vu m'a forcé à me défaire de cette image et à penser autrement. (réflexion d'un étudiant)

En somme, tant pour les élèves impliqués que pour les étudiants en formation à l'enseignement, les résultats de l'expérience montrent la richesse d’un apprentissage transformationnel ancré dans des réalités diverses et complémentaires. Les conclusions de notre étude permettent d'envisager un apprentissage plus global fait de liens entre les jeunes, les familles, les communautés et les étudiants universitaires. Pour se conformer aux principes énoncés par Cole dans Fifth Dimension Model (2006), évoquant l'importance de transposer les pratiques communautaires dans le contexte de l'école et de donner une voix aux élèves d'origines diverses, à leurs familles et à leurs communautés, il importe de travailler à briser les formes traditionnelles d'apprentissage pour les jeunes et pour les étudiants en formation à l'enseignement. 


\section{Références bibliographiques}

Cole, M. (et Distributed Literacy Consortium) (2006). The fifth dimension : An afterschool program built on diversity. New York : Russell Sage Foundation.

Gutiérrrez, K. (2014). Foreword: La Clase Magica: An enduring prototype of a new Latin@ Diaspora. Dans B. Flores, O. Vasquez et E. Clark (dir.), Generating transworld pedagogy (p. vii-xii). New York : Lexington Books.

McDonald, M., Bowman, M. et Brayko, K. (2013). Learning to see students: Opportunities to develop relational practices of teaching through community-based placements in teacher education. Teachers College Record, 115(4), 1-35.

Murrell, P. (2000). Community Teachers: A Conceptual Framework for Preparing Exemplary Urban Teachers, The Journal of Negro Education, 69(4), 338-348.

Tan, E. et Calabrese Barton, A. (2012). Empowering science and mathematics education in urban schools. Chicago : University of Chicago Press.

Zeichner, K. (2010a). Competition, economic rationalization, increased surveillance, and attacks on diversity: Neoliberalism and the transformation of teacher education in the US. Teaching and Teacher Education, 26, 1544-1552.

Zeichner, K. (2010b). Rethinking the connections between campus courses and field experiences in college- and university-based teacher education. Journal of Teacher Education, 61, 89-99. 